INFECTIVE

ENDOCARDITIS

\title{
Surgery for infective endocarditis
}

\section{M.R. Essop}

Division of Cardiology, Chris Hani Baragwanath Hospital, Soweto, Johannesburg

\section{Address for correspondence: \\ Professor M.R. Essop \\ Head, Division of Cardiology \\ PO Bertsham \\ Soweto}

2013

South Africa

Email:

essopmr@medicine.wits.ac.za

\section{INTRODUCTION}

Despite advances in antimicrobial chemotherapy, infective endocarditis (IE) remains a lethal disease with a case fatality rate of almost 40\%.(1,2) Surgery may be life saving, but the indication and timing for it are controversial. While much of our practice in cardiology is governed by the results of randomised control trials - the most rigorous test of the value of an intervention - this has not been possible when testing the role of surgery for IE. Reasons for this include logistical difficulties, the relatively low incidence of the disease in single centers and clinical heterogeneity. The European and American heart association guidelines provide a framework for surgical intervention in $\mathrm{IE}^{(3,4)}$ but it should be borne in mind that they are not based on level I evidence and that while they are useful, they do not replace mature and experienced clinical judgment.

\section{SURGERY FOR NATIVE MITRAL AND AORTIC}

\section{VALVE ENDOCARDITIS}

In essence, the need for surgery in patients with IE is determined by the interaction of three factors (I) Organism-related factors including virulence, propensity to cause local destruction and

\section{ABSTRACT}

The last decade has seen many changes in the landscape of infective endocarditis (IE) - ranging from the epidemiology, microbiology, diagnostic techniques and indication for antibiotic prophylaxis. Unfortunately, the role of surgery in the patient with endocarditis, including the indication, timing and type of surgery continues to be plagued by controversy. Although surgery has an important and established role in critically ill patients with endocarditis, the choice between medical therapy and surgery is often less clear cut and poses a major challenge to the physician treating this condition. The following article summarises some of these controversies including indications for surgery in native and prosthetic valve endocarditis, the timing of surgery, the choice of prostheses and issues surrounding anti-coagulation. SAHeart 2009; 6:84-89

susceptibility to antibiotics (2) Haemodynamic sequela related mainly to valvular insufficiency but including valve obstruction (so called Oslerian endocarditis) and rupture of fistulae into the systemic venous or pulmonary arterial circulation and (3) Systemic embolism and the potential for recurrence. Figure I depicts the variable contribution of each of these categories and serves to emphasise the fact that systemic embolism is the least important, and also the most controversial indication for surgery.

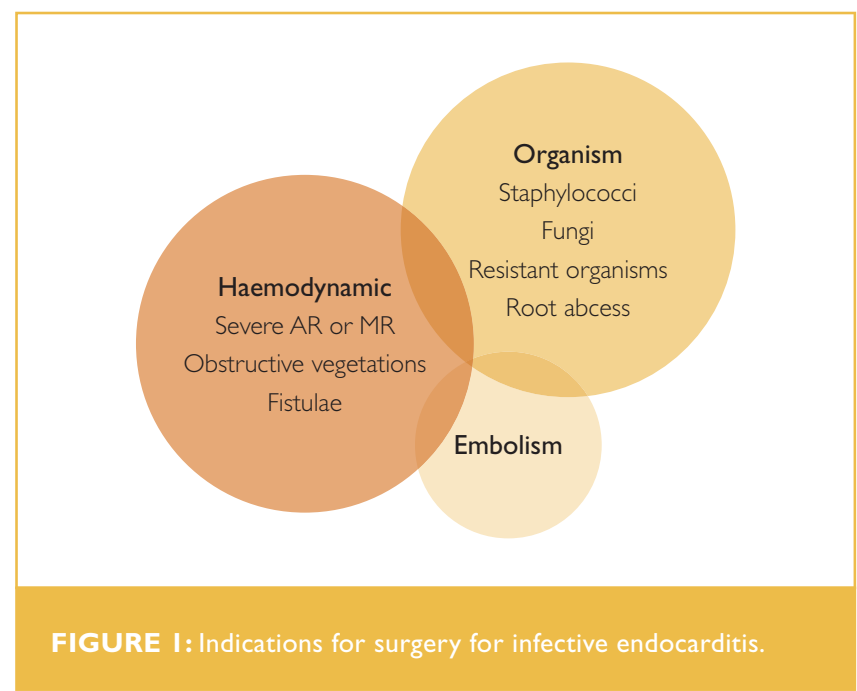


Factors related to the infecting organism

As a consequence of the capacity for extensive local tissue destruction or resistance to antibiotics, infection with certain organisms may predict a high likelihood that surgical intervention will be required.

Coagulase positive and negative staphylococcal infection is notorious for extensive tissue breakdown, local spread and a predilection for abcess and fistula formation. In many Western populations, S. Aureus is now the leading cause of nosocomial and community acquired health care associated endocarditis and the second commonest cause of non-health care associated IE. ${ }^{(5)}$ Apart from the intrinsic virulence of staphylococci, several other factors combine to increase the mortality (about 50\%) of medically treated staphyloccocal endocarditis.(6) These include older age with atypical presentation, presence of co-morbid disease and a rising incidence of resistance not only to the betalactams, but also to vancomycin. ${ }^{(7)}$ Infection extending beyond the valve leaflets occurs in 10-20\% of patients and affects the aortic valve more commonly than the mitral. Perivalvular abcess formation is an indication for urgent surgery. Persistent fever, appearance of new electrocardiographic conduction defects and pericarditis are clues to the presence of peri-aortic suppuration but lack sensitivity. The threshold to performing transesophageal echocardiography should be low and may allow earlier diagnosis (Figure 2).

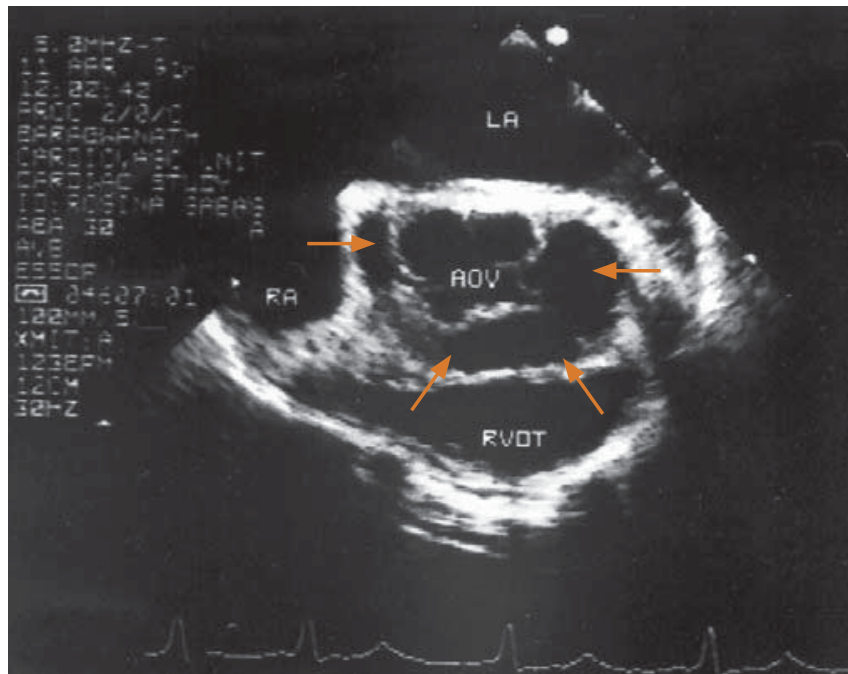

FIGURE 2: Transesophageal basal 4 chamber view showing circumferential perivalvular abcess (arrow). AV=aortic valve
Other organisms that are associated with a poor outcome with medical treatment include Streptococcus pneumonia, Group B streptococci, enterococci, gram negative bacilli, brucella and fungal endocarditis. The overall medical cure rate for fungal endocarditis is only $14 \%^{(8)}$ and is due in part to poor penetration of antifungal agents into the vegetation, the low toxic-therapeutic ratio of currently available agents and the common lack of fungicidal activity. A cure is virtually impossible without surgical intervention. The role of newer anti-fungal agents such as caspofungin and voriconazole is as yet undefined.

Irrespective of the organism, microbiologic or clinical evidence of persistent and uncontrolled infection despite appropriate antibiotics is a relative indication for surgery. Thus, surgery should be considered in a patient with persistently positive blood cultures or fever despite one week of antibiotic therapy after other aetiologies such as metastatic abcess, drug fever and confounding secondary infections have been ruled out.

Surgical intervention is also indicated for most patients with culture negative IE who exhibit evidence of persistent uncontrolled infection (fever for more than 7-10 days) without evidence of infection at another site.

Relapse of patients with native valve IE after adequate therapy may require surgical intervention, although a second course of antibiotics may be reasonable if there is no paravalvular suppuration and the causative organism is sensitive to antibiotics. It is important, however, to differentiate relapse from re-infection where a further course of antibiotics could be considered.

High levels of C-reactive protein after one week of antibiotic therapy have recently been found to predict serious complications and death from IE with an odds ratio of 10.3 in patients in the highest tertile versus the lowest tertile. ${ }^{(9)}$ While some studies have found C-reactive protein a useful predictor of need for surgery, this has not been a consistent finding.

\section{Haemodynamic factors}

The greatest benefits of surgery have been noted in patients with IE and heart failure that is almost always due to severe 
valvular insufficiency. Rarely, heart failure may be due to large obstructive vegetations. While antibiotic therapy is generally successful in curing infection, it can hardly be expected to make any impact on a valve that is already severely incompetent. Prior to the routine use of surgery, heart failure was responsible for almost $90 \%$ of deaths from IE. Mortality from medically treated heart failure in IE is $75 \%$ and can be reduced to $25 \%$ with surgical intervention. ${ }^{(10-13)}$ In a more recent report, Slater et al. documented their experience in 364 patients with IE seen over a 10 year period. ${ }^{(14)}$ Heart failure was the indication for surgery in 52\% of their patients and the presence of haemodynamic instability was an independent predictor of mortality. Surgery was associated with a significantly improved short and long term survival.

While most reports have confirmed the benefits of surgery in high risk patients with $I E$, a recent publication from the Mayo clinic failed to document any advantage, and suggested perhaps even an increased mortality in individuals having operative intervention. (15) Although the study used a propensity score to match surgical and non-surgical patients, it is arguable whether this technique is able to prevent completely the influence of selection bias

Acute aortic regurgitation constitutes a more precarious haemodynamic state than mitral regurgitation and is a reason to consider emergency surgery. It has always been our policy to submit these patients for surgery within 24-48 hours of starting antibiotics with excellent long term results. ${ }^{(16)}$ Echocardiography is useful to identify patients with acute severe aortic regurgitation ${ }^{(17)}$ due to IE by demonstrating premature closure of the mitral valve on M-Mode (Figure 3a) and diastolic mitral regurgitation with combined M-Mode and color flow imaging or pulsed Doppler (Figure 3b). It is important that other causes of heart failure such as sepsis, anaemia and pre-existing left ventricular dysfunction be ruled out before implicating valvular regurgitation as the mechanism.

A more controversial indication for surgery is the patient with severe mitral or aortic regurgitation but without clinical heart
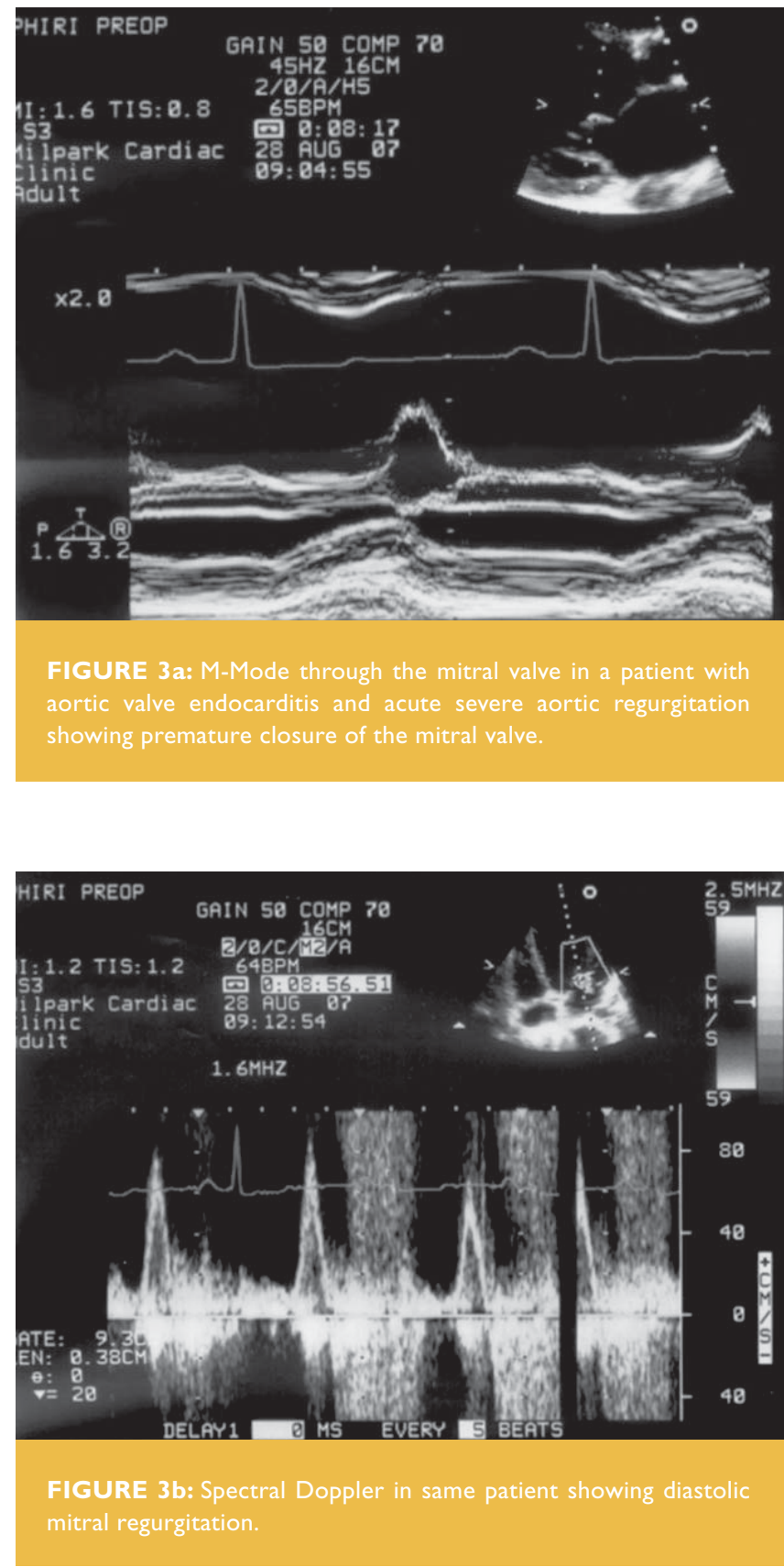

failure. While the ACC/AHA guidelines list this as a relative indication for surgery, ${ }^{(4)}$ the absolute benefits may not be evident especially where operative intervention entails replacement with a mechanical prosthetic valve with the attendant risks of prosthetic valve endocarditis, thrombosis, embolism and anticoagulant related bleeding. We therefore believe that these patients should be closely monitored and the need for surgery dictated by concomitant factors which require surgery in their own right. 


\section{Embolic complications}

Clinical systemic embolisation is seen in 10-35\% of patients although occult events may occur more frequently. ${ }^{(18)}$ The significance of echocardiographically detected vegetations as a risk factor for systemic embolism is controversial. The presence of large vegetations does, however, appear to be predictive of increased morbidity, need for surgery and mortality.

The best treatment for echocardiographically detected vegetations is not surgery, but prompt and appropriate antibiotic therapy following which clinically evident embolisation is unusual. The literature, however, is replete with studies examining the value of a variety of echocardiographic characteristics of vegetations such as mobility and size which may predict risk of embolisation and need for surgery. Despite a strong direct association between vegetation size and risk for thromboembolism, not all patients with large vegetations invariably have a stroke and conversely, some patients with relatively small vegetations suffer disabling events. Non-vegetation related predictors of embolism include age, staphylococcal infection and high CRP. Thus, in general, vegetation size alone should not be a reason for surgical intervention but in conjunction with other factors such as persistent sepsis or valve dysfunction, may lower the threshold for operation.

Recurrent systemic embolism despite appropriate antibiotic therapy is an indication for surgery, but there is no consensus on what the definition of recurrent should be. Most authors would concur that two or more episodes occurring during antibiotic therapy represents a reasonable indication for surgery.(19)

\section{SURGERY FOR PROSTHETIC VALVE}

\section{ENDOCARDITIS}

By its very nature, prosthetic valve endocarditis (PVE) is a more serious disease than native valve IE with an overall mortality of almost 33\%. Early PVE (defined as occurring within 60 days of surgery) carries a much higher mortality than late PVE, mainly because of a greater incidence of coagulase positive and negative staphylococcal infection. As with native valve IE, heart failure and staphylococcal infection are independent predictors of mortality ${ }^{(20)}$ but there are several complications that are unique to PVE. These include valve dehiscence, paraprosthetic ring leaks and mechanical dysfunction of the prosthesis. While transoesophageal echocardiography is important in the diagnosis and management of $\mid E^{(2 \mid)}$ it finds particular value, and is mandatory, in patients with PVE in whom vegetations, abcesses, fistulae and ring leaks may not be visualised by any other technique.

A medical cure is far less likely with PVE. The recommendations for surgery are the same for native valve IE but additional indications include prosthetic valve dysfunction and an unstable prosthesis, both of which constitute an indication for urgent operative intervention.

Unfortunately, for a number of reasons, the risks of surgery are also higher for PVE than for native valve IE. These include the additional risk associated with re-operation, more aggressive perivalvular suppuration requiring more complex surgery and the inability to perform valve repair as with native valve IE.

\section{TIMING OF SURGERY}

If the indications for surgery are controversial, the decision as to when to operate in patients with IE may be even more difficult, requiring greater clinical judgment. Many of the complications of IE are sudden, catastrophic and often fatal, and the decision not to have operated earlier is not infrequently regretted. On the other hand, physicians frequently prefer to optimise patients medically and institute antibiotic therapy in order to sterilise the area of planned surgical intervention. Contrary to popular belief, however, surgery is not contraindicated in the presence of active infection and in fact may be life saving. Critically ill patients in whom antimicrobial therapy has not yet been initiated, should be commenced immediately on broad spectrum antibiotics after blood cultures have been drawn and monitored intensively thereafter. Any deterioration, or failure to improve after 24-48 hours of treatment requires prompt surgical intervention providing the previously discussed indications are met. This approach requires a multi-disciplinary approach with early and close interaction between cardiologist, cardiothoracic surgeon 
and microbiologist. The indications for surgery according to urgency are listed in Table I.

TABLE I: Indications for surgery in infective endocarditis according to urgency

Emergency surgery (<24 hours)
Aortic regurgitation with acute severe heart failure
Rupture of a sinus of Valsalva into adjacent structures
Prosthetic valve malfunction
Prosthetic valve dehiscence
Urgent surgery (24-72 hours)
Perivalvular abcess
Severe mitral or aortic regurgitation with heart failure
Non urgent surgery
Persistent fever despite appropriate antibiotics
Recurrent cerebral emboli
Resistant organisms - Staphylococci, Fungi
Relapse after previous antibiotic treatment

\section{ASPECTS OF SURGERY FOR INFECTIVE}

\section{ENDOCARDITIS}

\section{Surgical technique and choice of prosthesis}

Details of operative approach are better covered in surgical texts. A fundamental principle of surgery, however, is radical debridement and extirpation of all infected tissue so that the new valve may be implanted on strong and healthy tissue. Surgical reconstruction of the aortic root for peri-aortic suppuration, sometimes with almost complete aorto-left ventricular discontinuity, poses a tremendous challenge, but equally challenging is resection of abcesses located in the posterior mitral annulus and in the aorto-mitral curtain. ${ }^{(22)}$

Aortic valve repair is rarely an option and the choice of prosthesis in this position is a matter of some debate. However, most surgeons agree that scrupulous removal of all infected tissue is probably more important than the type of valve selected. (22) Homografts may be more resistant to re-infection but their main advantage seems to be that they are ideally suited to reconstructing the aortic root, ease of handling and the ability to use the remnant of the anterior mitral valve for patching defects created by resection of abcesses. ${ }^{(22)}$ Although the risk of recurrent IE appears to be similar for mechanical and bioprosthetic valves, ${ }^{(23)}$ the Toronto group prefer mechanical valves because of easier implantation. (22)

As for surgery for mitral regurgitation not associated with IE, repair is preferable to replacement provided the surgeon can guarantee with a high degree of probability that the patient will not be left with anything more than mild residual regurgitation. Not infrequently, infection may spread onto the chordae and papillary muscle and it is imperative that this be debrided.

Importantly, surgeons need to be reminded that all resected tissue be submitted for culture and histology. Following surgery, antibiotics should be continued for the planned total duration of 4-6 weeks, but where the valve culture is positive, the ESC guidelines recommend another full course of antimicrobial therapy. ${ }^{(3)}$

Surgery for infective endocarditis complicated by stroke The decision regarding cardiac surgery in the patient with IE already complicated by a stroke may be particularly difficult. Apart from the ethical issue of submitting a patient for a major cardiac procedure who may have suffered substantial neurologic damage from which recovery may be unlikely, the risk benefit ratio of converting an ischaemic infarct into a haemorrhagic one or extending an intracerebral bleed during cardiopulmonary bypass have to be carefully considered. Recommendations regarding the optimal timing of surgery after cerebral infarction range from immediate, (24) if the reason for cardiac surgery is compelling, up to 4 weeks. ${ }^{(25)}$ An additional factor to consider in the timing of surgery is the continued presence of a large mobile vegetation with a high likelihood of further embolism. In the Japanese study ${ }^{(25)}$ of 181 patients with IE and stroke, $44 \%$ had further neurologic deterioration if a non-haemorrhagic cerebral event had occurred within 7 days of surgery, compared with only $2.3 \%$ experiencing deterioration if surgery was delayed by 4 weeks. In haemorrhagic stroke, the risk persisted for up to 4 weeks. The consensus of opinion seems to be to delay surgery up to 2 weeks for a non- 
haemorrhagic cerebral event and up to a month for an intracerebral bleed. Patients with an intracerebral bleed should have conventional cerebral or magnetic resonance angiography in order to detect ongoing bleeding from a ruptured aneurysm. If present, this should be corrected prior to cardiac surgery.

\section{Anticoagulation issues}

Patients with PVE on chronic Warfarin anticoagulation should be switched to unfractionated or low molecular weight heparin if surgery is anticipated. If a neurologic event has already occurred in a patient on chronic warfarin therapy, it is important to document by means of brain CT or MRI the presence of a bleed. If a bleed is confirmed, all anticoagulation should be stopped since the risk of further intracerebral bleeding outweighs the risk of prosthetic valve thrombosis, but the duration of cessation should be individualised for each patient.

\section{CONCLUSION}

The fact that the role of surgery for IE is surrounded by some controversy should not detract from the important contribution it makes to the management of these patients. In patients with acute severe aortic regurgitation, refractory heart failure related to significant mitral or aortic regurgitation and those with advanced and locally destructive complications, surgery may be life saving.

\section{REFERENCES}

I. Bashore TM, Cabell C, Fowler V. Update on infective endocarditis. Curr Prob Cardiol 2006;31:274-352

2. Koegelenberg CFN, Doubell AF, Orth $H$, et al. Infective endocarditis in the Western Cape Province of South Africa: a three year prospective study. Q J Med 2003;96:217-225.

3. Horskotte D, Follath F, Gutschik E, et al. Guidelines on prevention, diagnosis and treatment of infective endocarditis. The task force on infective endocarditis of the European Society of Cardiology. Eur Heart J 2004;00: I-37.

4. Baddour LM, Wilson WR, Bayer AS, et al. Infective endocarditis: diagnosis, antimicrobial therapy and management of complications: a statement for healthcare professionals from the Committee on Rheumatic Fever, Endocarditis and Kawasaki Disease, Council on Cardiovascular Disease in the Young and the Council on Clinical Cardiology, Stroke, and Cardiovascular Surgery and Anaesthesia, American Heart Association - Executive Summary. Circulation 2005; 1 11:3167-84.
5. Fowler VG, Miro JM, Hoen B, et al. Staphylococcus aureus endocarditis: A consequence of medical progress. JAMA 2005;293:3012-21.

6. Bouza E, Menasalvas A, Munoz P, et al. Infective endocarditis : a prospective study at the end of the 20th century-new predisposing conditions, new etiologic agents, and still a high mortality. Medicine (Baltimore) 2001;80:298-307.

7. Drees M, Boucher $\mathrm{H}$. New agents for Staphylococcus aureus endocarditis. Curr Opin Infec Dis 2006; 19:544-50.

8. Rubenstein E, Noriega ER, Simberkoff MS, et al. Tissue penetration of Amphotericin B in Candida endocarditis. Chest 1974:66:376-80.

9. Verhagen DWM, Hermanides J, Korevaar JC, et al. Prognostic value of serial C-reactive protein measurements in left-sided native valve endocarditis. Arch Intern Med 2008; 1 68(3):302-7.

10. Alsip SG, Blackstone EH, Kirklin JW, et al. Indications for cardiac surgery in patients with active infective endocarditis. Am J Med 1985;78(Suppl 6B): 138-46.

II. Mullany C], Mclsaacs Al, Rowe MH, et al. The surgical treatment of infective endocarditis. World J Surg 1989;13:132-39.

12. Al Jubair K, Al Fagih M, Ashmeg A, et al. Cardiac operations during active endocarditis. JThorac Cardiovasc Surg 1992; 104:487-95.

13. Larbalestier RI, Kinchla NM, Aranki SF, et al. Acute bacterial endocarditis: optimising surgical results. Circulation 1992;86(Suppl |I): | 168-76.

14. Slater MS, Komanapalli CB, Tripathy $U$, et al. Treatment of endocarditis: a decade of experience. Ann Thorac Surg 2007;83:2074-80.

15. Tleyjeh IM, Ghomrawi HMK, Steckelberg JM, et al. The impact of valve surgery on 6 month mortality in left-sided infective endocarditis. Circulation 2007; 115:1721-1728.

16. Middlemost S, Wisenbaugh T, Meyerowitz C, et al. A case for early surgery in native left-sided endocarditis complicated by heart failure:results in 203 patients. J Am Coll Cardiol 1991;18:663-71.

17. Sareli P, Klein HO, Schamroth CL, et al. Contribution of echocardiography and immediate surgery to the management of severe aortic regurgitation from active infective endocarditis. Am J Cardiol 1986;57:413-18.

18. Fabri J, Issa VS, Pomerantzeff PM, et al. Time-related distribution, risk factors and prognostic influence of embolism in patients with left-sided endocardtis. Int J Cardiol 2006; I 1 0:3344 I.

19. Bayer AS, Bolger AF, Taubert KA, et al. Diagnosis and management of infective endocarditis and its complications. Circulation 1998;98:2936-48.

20. Habib G, Tribouilloy C, Thuny F, et al. Prosthetic valve endocarditis: who needs surgery? A multi-center study of 104 cases. Heart 2005;91:254-61.

21. Essop MR. Transesophageal echocardiography for the diagnosis of infective endocarditis - a standard for the nineties? (editorial). American Heart Journal 1995; 1 30:402-404.

22. David TE, Gavra G, Feindel CM, et al. Surgical treatment of active infective endocarditis: a continued challenge. JThorac Cardiovasc Surg 2007; 133:144-9.

23. Moon MR, Miller DC, Moore KA, et al. Treatment of endocarditis with valve replacement: the question of tissue versus mechanical prosthesis. Ann Thorac Surg 200।;7|:| |64-7|.

24. Ruttmann E, Willeit J, Ulmer $\mathrm{H}$, et al. Neurological outcome of septic cardioembolic stroke after infective endocarditis. Stroke 2006;37:2094-99.

25. Eishi K, Kawazoe K, Kuriyama Y, et al. Surgical management of infective endocarditis associated with cerebral complications. Multi-center retrospective study in Japan. JThorac Cardiovasc Surg 1995; I 1 0: 1745-63. 